\title{
Spatial distribution of attentional inhibition is not altered in healthy aging
}

\author{
Linda K. Langley • Nora D. Gayzur • Alyson L. Saville • \\ Shanna L. Morlock • Angela G. Bagne
}

Published online: 17 November 2010

(C) Psychonomic Society, Inc. 2010

\begin{abstract}
Inhibition of return (IOR) is a phenomenon of attentional orienting that is indexed by slower responses to targets presented at previously attended locations. The purpose of this study was to examine adult age differences in the distribution of IOR to multiple locations. In three experiments, young adults (ages 18-30 years) and older adults (ages 60-87 years) completed an IOR task that varied in the number of simultaneous onset cues (one to seven) and the number of display locations (four or eight). Analyses were conducted to explore whether IOR patterns were most consistent with limited inhibitory resources, with regional distribution of inhibition, or with vector averaging of cues. The IOR effects were most consistent with vector averaging, such that multiple cues initiated a directional gradient of inhibition centered on the average direction of the cues. The IOR patterns varied minimally with age, consistent with the conclusion that older adults and young adults distributed inhibition in a similar manner.
\end{abstract}

Keywords Inhibition of return - Aging - Spatial distribution . Vector averaging $\cdot$ Working memory $\cdot$ Multiple cues

\section{Introduction}

Inhibition of return (IOR) is a delayed response to items presented at previously attended locations (Posner \&

Angela Bagne is now at Minnesota State Community and Technical College, Moorhead, MN.

L. K. Langley $(\bowtie) \cdot$ N. D. Gayzur · A. L. Saville

S. L. Morlock · A. G. Bagne

Department of Psychology, North Dakota State University,

Dept 2765, P.O. Box 6050, Fargo, ND 58108-6050, USA

e-mail: linda.langley@ndsu.edu
Cohen, 1984). Spatial cues initially serve to facilitate orienting to a location. For instance, if a target item is presented within $100 \mathrm{~ms}$ of an abrupt onset cue, observers detect the target more quickly at the cued location than at an uncued location, presumably due to a reflexive shift of attention towards the source of stimulation. However, if attention is disengaged from the cued location (exogenously by a cue presented elsewhere, or endogenously because the cue is uninformative and is not followed immediately by a target), item detection is slowed at the cued location compared to a target presented at an uncued location. This inhibitory effect is thought to reduce re-examination of searched locations and to promote novelty in visual exploration (see Klein, 2000, for a comprehensive overview of IOR).

IOR was initially observed following a single peripheral cue in a two-location display (Posner \& Cohen, 1984). If IOR is to facilitate foraging in real world environments, then it should inhibit the return of attention to multiple locations (Klein, 1988). Posner and Cohen found that, when both peripheral locations were simultaneously cued, responses to targets at either location were just as slow as responses to targets following a single cue, which they interpreted to indicate that both locations were successfully inhibited in the double-cue condition. In contrast, Maylor (1985, Experiment 3) found significant but reduced inhibition in double-cued trials, suggesting that simultaneous stimulation of more than one location resulted in reduced inhibition at a particular location. With only two locations, there was no uncued condition with which to assess inhibitory effects independent of alerting effects (i.e., two cues may have had a larger alerting or preparatory effect than a single cue). Wright and Richard (1996) presented up to four simultaneous cues in an eightlocation array and compared responses on cued and uncued 
trials. They found IOR for as many as four cues, and IOR did not decline in magnitude as cue number increased. The spatial distribution of IOR has been investigated using sequential as well as simultaneous cuing. Inhibition has been found to exist at up to five sequentially-cued locations, with inhibition being greatest for the most recently cued locations, consistent with a temporallybased decline in IOR (Birmingham \& Pratt, 2005; Dodd, Castel, \& Pratt, 2003; Snyder \& Kingstone, 2000). In sum, across cuing paradigms, the findings suggest that inhibition can be associated with at least four or five locations.

A goal of the present study was to compare three explanations for how IOR is distributed to multiple locations: (1) limited inhibitory resources, (2) regional distribution of IOR, and (3) a gradient of inhibition corresponding to the vector average of cues. A resources account of IOR posits that working memory is involved in "tagging" the locations of attended objects and in allocating inhibition to those locations (Castel, Pratt, \& Craik, 2003). With limits to working memory, it follows that there are limits to the number of objects that can be simultaneously tagged and inhibited. Once resource limits are exceeded, inhibition associated with a particular object diminishes.

According to a regional distribution account of IOR (Abrams \& Pratt, 1996), inhibition can be diffused within a region, such that when cued locations are adjacently located, observers partition the visual space into a cued region and an uncued region. What might be interpreted as IOR for separate locations might actually be IOR for one spatially diffuse region. Consistent with this interpretation, Abrams and Pratt found that when two of four potential target locations were sequentially cued, only the most recently cued location was inhibited when the cued locations were non-adjacent, but both locations were inhibited when the cued locations were adjacent. In contrast to these findings, Danziger, Kingstone, and Snyder (1998) found evidence for IOR at non-adjacent locations. They eliminated all trials in which cues were presented at adjacent locations and still found IOR for two and three sequentially-presented cues. In addition, there was little difference in the magnitude of IOR for adjacent and separated cues. Thus, although it appears that IOR can be associated with multiple discrete locations, at least in a sequential paradigm, there is some evidence that there is a spatially diffuse type of inhibition that leads contiguous locations to receive supplemental inhibitory processing.

Instead of IOR being distributed discretely to cued locations, Klein, Christie, and Morris (2005) proposed that IOR following simultaneous cues is distributed as a gradient. Past research had demonstrated a gradient pattern of IOR following single cues (Bennett \& Pratt, 2001; Maylor \& Hockey, 1985; Pratt, Spalek, \& Bradshaw, 1999). Response times were slowest when the target was presented at the same location as the cue and gradually decreased the further removed in space the target was from the cue. Klein and colleagues argued that inhibition following multiple cues was not parceled out to the cued locations; instead, the cues activated an orienting response that was directed toward the net vector of the cues. Inhibition was likewise directed toward the net vector, with a gradient of diminishing inhibition spreading from that point. For example, if two cues were presented within a circular array, one at $0^{\circ}$ (the top of the array) and the other at $90^{\circ}$ (the right side of the array), the orienting response and subsequent IOR would be strongest at $45^{\circ}$ (the upper right corner; the average direction of the two cues) and would gradually diminish in strength the further removed the target was from that location. Thus, IOR could be greater at an uncued location $\left(45^{\circ}\right)$ than at the cued locations $\left(0\right.$ or $\left.90^{\circ}\right)$ if the uncued location fell at or near the average direction of the cues.

With up to four cues in an eight-location circular array, Klein and colleagues (2005) found that with the typical measure of IOR (slower responses to targets at cued than uncued locations, averaged across locations), IOR did not vary significantly in magnitude following one, two, three, or four cues, replicating the Wright and Richard (1996) pattern. However, when response times were examined as a function of the distance between the target and the average direction of the cues, a gradient pattern emerged that mimicked the gradient pattern observed following single cues. Furthermore, response times following multiple cues did not vary significantly in the cued and uncued conditions, supporting the conclusion that inhibition was distributed based on the vector average of cues rather than by local stimulation from individual cues. Finally, on multiple-cue trials in which the cues' center of gravity fell in the center of the array (for an example, see the two-cue arrangement of Fig. 1, below) rather than at or near an array location, response times did not vary between cued and uncued trials, suggesting that participants maintained attention at the center of the array rather than directing it toward the cued locations. Together, the findings were consistent with the conclusion that inhibition was distributed based on the average orienting direction of multiple cues.

To compare the three accounts of multiple-location IOR, in the present study we tested participants on a simultaneous-cuing IOR task that varied in the number of onset cues and the number of array locations. In Experiment 1 , we used a four-location task with one, two, or three cues. In Experiment 2, we used an eight-location array with one, three, five, or seven cues. Finally, in Experiment 3, participants were presented with one to four cues in an eight-location array. To test the resources explanation, we determined the magnitude of IOR as a function of cue 


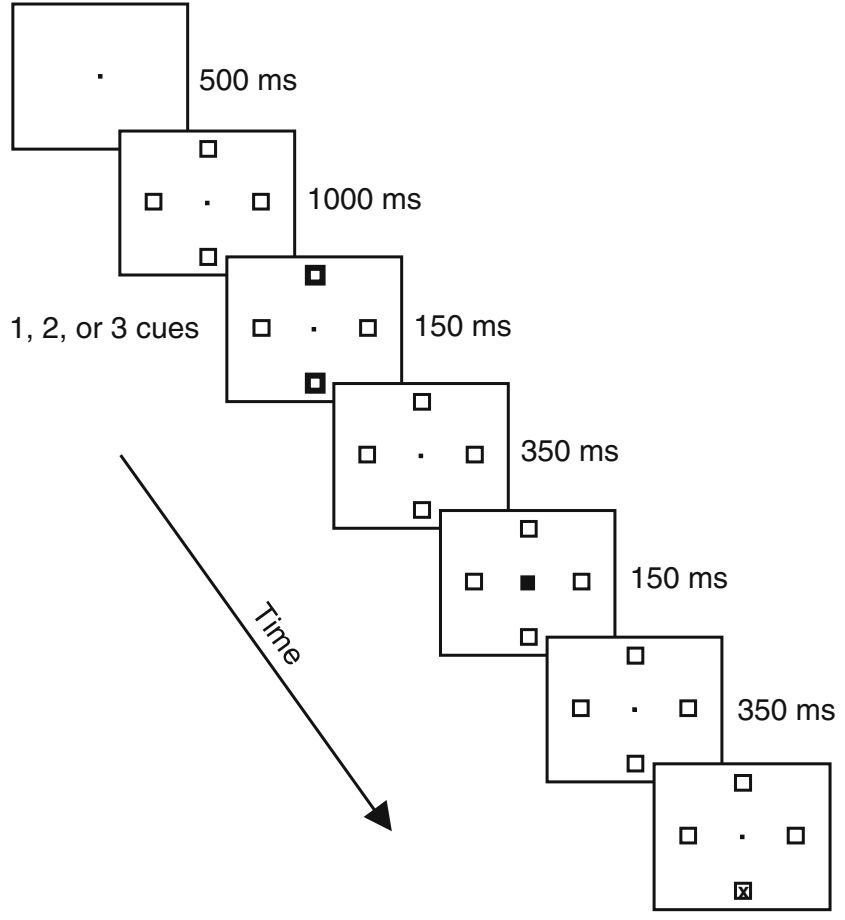

Fig. 1 Trial sequence for Experiment 1. White stimuli were presented against a black background. Stimuli are not scaled to size. One, two, or three cues were presented within a four-location display

number. If IOR could be associated with only a limited number of locations, then as the number of cues increased, the magnitude of IOR should have decreased. To determine whether IOR was distributed in a regional manner, we compared the magnitude of IOR for cues that were presented at adjacent locations versus cues that were presented at spatially discrete locations. If IOR were associated with multiple locations only when these locations were contiguous (and thus processed as a region), then IOR effects would be greater for adjacent cues than for separated cues. Finally, we repeated the analyses of Klein et al. (2005) to test a gradient explanation of IOR. If IOR were distributed as a gradient, then we would expect response times to diminish as the distance between the target and the average direction of the cues increased. Furthermore, the responses to targets should have been influenced by the directional gradient rather than by local stimulation from cued locations, so for multiple cues with a substantial average vector, responses should have been similar for cued and uncued trials. On trials in which there was no strong directional vector due to the spatial distribution of cues, IOR should have been minimal.

The second goal of the present study was to determine whether there are adult age differences in the spatial distribution of IOR. Older adults have difficulty, relative to young adults, efficiently locating a target from an array of distracting items (Foster, Behrmann, \& Stuss, 1995;
Humphrey \& Kramer, 1997). Explanations for age-related changes in visual search have included reduced ability to adjust the attentional focal area (Greenwood \& Parasuraman, 2004; Rösler, Mapstone, Hays-Wicklund, Gitelman, \& Weintraub, 2005), difficulty with strategically setting search parameters (Gottlob, 2006), and slowed disengagement from searched locations (D'Aloisio \& Klein, 1990; Klein, 2005; Rösler et al., 2005). Alternatively, search inefficiency may be explained, at least in part, by a premature return of attention to searched locations. Although studies have reported age equivalencies in IOR for single locations (Faust \& Balota, 1997; Hartley \& Kieley, 1995; Langley, Vivas, Fuentes, \& Bagne, 2005), older adults may have difficulty inhibiting reorienting to multiple locations. One study to date has examined age differences in multiple-location IOR. Pratt and Chasteen (2007) found that, following sequential cues, young and older adults maintained inhibition at up to five locations, with no age differences in the magnitude of IOR. The authors concluded that the form of visuospatial memory required to tag multiple locations was relatively resistant to age-related changes.

The means by which inhibition is distributed to multiple cues impacts interpretation of age-related differences in IOR. If a limited resources explanation best accounts for IOR distribution, then we might observe that, for older adults, declines in IOR as cue number increases are steeper, consistent with an age-related reduction in inhibitory resources. If a regional explanation best accounts for IOR distribution, it might be the case that older adults are less able to process adjacent locations as an inhibited region, thus leading to reduced differences in IOR between adjacent and separated locations. If vector averaging best explains multiple-location IOR, then the vector averaging processes associated with orienting may change with age, influencing the distribution of inhibition.

\section{Experiment 1}

In Experiment 1, we presented one, two, or three cues simultaneously in a four-location array. To examine the manner in which the two age groups allocated inhibition, we analyzed the data to compare limited resources, regional inhibition, and vector averaging accounts of IOR.

\section{Method}

Participants Participants were 20 young adults (13 women, 7 men) in the age range of 18-22 years and 20 older adults (15 women, 5 men) in the age range of 61-78 years. Demographic and screening data are reported in Table 1. Young adults were students in psychology classes at North Dakota State University who received course credit for 
Table 1 Participant characteristics for Experiments 1, 2, and 3

\begin{tabular}{|c|c|c|c|c|c|c|c|c|c|c|c|c|}
\hline & \multicolumn{4}{|c|}{ Experiment 1} & \multicolumn{4}{|c|}{ Experiment 2} & \multicolumn{4}{|c|}{ Experiment 3} \\
\hline & \multicolumn{2}{|l|}{ Mean } & \multicolumn{2}{|l|}{ SD } & \multicolumn{2}{|l|}{ Mean } & \multicolumn{2}{|l|}{ SD } & \multicolumn{2}{|l|}{ Mean } & \multicolumn{2}{|l|}{ SD } \\
\hline & OA & YA & OA & YA & OA & YA & OA & YA & OA & YA & $\mathrm{OA}$ & YA \\
\hline Age (yrs) & $68.4 *$ & 19.2 & 5.5 & 1.1 & $71.5^{*}$ & 19.4 & 6.6 & 2.3 & $69.0 *$ & 19.6 & 5.7 & 1.8 \\
\hline Education (yrs) & $15.0^{*}$ & 13.0 & 2.2 & 1.0 & $14.6^{*}$ & 13.4 & 2.6 & 0.8 & $15.0^{*}$ & 13.7 & 2.6 & 1.3 \\
\hline WASI vocabulary (80 max) & $64.9 *$ & 56.6 & 7.8 & 3.5 & $64.5^{*}$ & 59.6 & 8.5 & 6.4 & $65.3 *$ & 56.1 & 7.8 & 5.3 \\
\hline Snellen acuity $(20 / \ldots)$ & $23.7 *$ & 14.7 & 7.0 & 2.1 & $24.6^{*}$ & 17.3 & 8.5 & 5.9 & $24.2 *$ & 15.6 & 7.1 & 3.5 \\
\hline MMSE (30 max) & 29.1 & 29.5 & 1.1 & 0.6 & 29.6 & 29.4 & 0.6 & 0.8 & 29.4 & 29.7 & 0.7 & 0.5 \\
\hline GDS (30 max) & 1.1 & 1.8 & 2.0 & 2.2 & 2.1 & 1.6 & 2.3 & 2.0 & 1.6 & 2.1 & 2.1 & 1.8 \\
\hline
\end{tabular}

SD standard deviation, $O A$ older adult group, YA young adult group, WASI Wechsler Abbreviated Scale of Intelligence (Wechsler, 1999)

Maximum score on the vocabulary subscale is 80 points, with a higher score indicating better performance

Snellen acuity denominator of the Snellen fraction for corrected near vision. A smaller number indicates better vision

MMSE Mini Mental State Examination. Maximum score is 30 points, with a higher score indicating better performance

GDS Geriatric Depression Scale. Maximum score is 30, with a higher score indicating greater depression.

*Older adults' mean scores differed from those of young adults according to an independent $t$ test, $p<.05$

participating. Older adults were residents of the local community who received $\$ 15$ for participating. All participants were fluent in English and had obtained at least a high school education. Based on self-report (Christensen, Moye, Armson, \& Kern, 1992), all participants were free of medical conditions that could interfere with normal cognitive functioning (e.g., stroke, psychiatric illness, dementia, history of head injury). All participants scored 9 points or lower on the Geriatric Depression Scale (GDS; Yesavage et al., 1982), indicating minimal depressive symptoms, and all participants scored 26 points or higher on the MiniMental State Examination (MMSE; Folstein, Folstein, \& McHugh, 1975), indicating no apparent signs of significant cognitive impairment. Included participants had near visual acuity scores of $20 / 40$ or better.

Materials and stimuli The experiment was generated using E-Prime version 1.1 (Psychology Software Tools, Pittsburgh, PA, USA). Stimuli were displayed on a 17inch $(\mathrm{c} .43 .2-\mathrm{cm})$ color monitor controlled by a PC computer with a Pentium 4 processor. The stimulus display consisted of four white outlined boxes that were presented on a black background and that were arranged immediately above, below, to the right, and to the left of a central fixation point (a small white-filled square $0.43^{\circ}$ in width and height). The boxes, with visual angles of $1.79^{\circ}$ in width and height, were $7.15^{\circ}$ from the fixation point center-to-center. The target stimulus was a red $\mathrm{X}$ that was $0.72^{\circ}$ in width and height, presented in Arial font. Responses were made on a PST Serial Response Box (Psychology Software Tools), and a chin rest maintained viewing distance at $40 \mathrm{~cm}$.
Procedure Participants completed the screening battery and computer task during a $1-1 / 2 \mathrm{~h}$ testing session. The experimenter explained the computer task with scripted verbal instructions, and a screen recapped the instructions at the beginning of each block. After 15 practice trials, participants completed 720 test trials randomly divided into nine blocks of 80 trials. The trial sequence is shown in Fig. 1. Each trial began with the fixation point presented for $500 \mathrm{~ms}$, after which the four white outlined boxes were added to the display and remained on the screen for the duration of the trial. One, two, or three of the boxes were cued 1,000-ms later by increasing the thickness of the box outline (from 1 point to 5 points) for $150 \mathrm{~ms}$. After an interval of $350 \mathrm{~ms}$, the central fixation point was cued for $150 \mathrm{~ms}$ by increasing the size of the square (to $0.72^{\circ}$ squared). The target $\mathrm{X}$ was presented in the center of one of the peripheral boxes after another $350 \mathrm{~ms}$ and remained on the screen for $5 \mathrm{~s}$ or until the participant responded. The target was equally likely to appear at the four array locations, regardless of cue location (Klein et al., 2005). The cue-to-target stimulus onset asynchrony (SOA) between the initial cue and the target was $1,000 \mathrm{~ms}$. Participants were told to maintain gaze on the fixation point throughout the trial and to press the response key as soon as the target was presented. Eye movements were not monitored. To reduce anticipatory responses, catch trials (in which the target was not presented) were presented on $20 \%$ of trials (144 total). There were 48 cued trials and 144 uncued trials for the one-cue condition, 96 cued trials and 96 uncued trials for the two-cue condition, and 144 cued trials and 48 uncued trials for the three-cue condition. If participants 
responded before the target was presented, or if they failed to respond within $5 \mathrm{~s}$ of target presentation, they heard a brief error tone. Participants were encouraged to take breaks between test blocks.

Results

Limited resources analysis Mean reaction times (RTs), IOR difference scores (cued RT minus uncued RT), and error rates for Experiment 1 are reported in Table 2. Error trials (less than $1 \%$ for each age group) were removed from the RT analysis. In addition, RTs that were less than $150 \mathrm{ms,}$ more than $2,000 \mathrm{~ms}$, or more than 2.5 standard deviations away from a participant's condition mean were eliminated. Mean RTs were submitted to a $2 \times 3 \times 2$ mixed analysis of variance (ANOVA) with age group (young adults and older adults) as the between-subjects factor and cue number $(1,2$, and 3 cues) and target location (cued and uncued) as the within-subjects factors. All main effects were significant. Older adults were slower than young adults (404 and $316 \mathrm{~ms}$, respectively), $F(1,38)=32.80, p<.0001$, RT decreased as the number of cues increased $(366,359$, and $354 \mathrm{~ms}$ for 1,2 , and 3 cues, respectively), $F(2,76)=28.93$, $p<.0001$, and IOR was demonstrated by slower RTs to targets at cued than at uncued locations $(368$ and $351 \mathrm{~ms}$, respectively), $F(1,38)=152.45, p<.0001$. None of the interactions were significant, $p \mathrm{~s}>.20$, indicating that IOR did not vary substantially in magnitude as a function of age group or number of cues.

Table 2 Mean reaction times (ms) and error rates (\%) for Experiment 1

\begin{tabular}{|c|c|c|c|c|c|c|}
\hline \multirow[b]{2}{*}{ Number of cues } & \multicolumn{3}{|c|}{ Older adults } & \multicolumn{3}{|c|}{ Young adults } \\
\hline & 1 & 2 & 3 & 1 & 2 & 3 \\
\hline \multicolumn{7}{|l|}{ RT means } \\
\hline Cued & 421 & 411 & 407 & 328 & 325 & 319 \\
\hline Uncued & 402 & 393 & 389 & 314 & 308 & 302 \\
\hline IOR & $19^{*}$ & $18^{*}$ & $18^{*}$ & $14^{*}$ & $17^{*}$ & $17^{*}$ \\
\hline \multicolumn{7}{|l|}{ RT SDs } \\
\hline Cued & 52 & 45 & 47 & 47 & 56 & 51 \\
\hline Uncued & 49 & 51 & 48 & 45 & 54 & 48 \\
\hline IOR & 18 & 14 & 11 & 9 & 7 & 16 \\
\hline \multicolumn{7}{|l|}{ Error rates } \\
\hline Cued & 1.4 & 1.0 & 0.7 & 0.4 & 0.9 & 0.9 \\
\hline Uncued & 1.0 & 1.0 & 0.7 & 0.9 & 1.0 & 1.3 \\
\hline
\end{tabular}

$R T$ Reaction time, IOR inhibition of return difference score (cued RT minus uncued RT), $S D$ standard deviation

*IOR scores were significantly greater than zero according to a onesample $t$ test, $p<.05$
Regional inhibition analysis The above analyses are consistent with the possibility that young adults and older adults were able to maintain inhibition at multiple distinct locations. However, it is possible that neighboring locations were treated as a unitary cued region rather than separately cued locations (Abrams \& Pratt, 1996). To examine this possibility, we classified two-cue trials as having adjacent cues if the two cues were at contiguous locations (e.g., top and right; $67 \%$ of trials) and as having separated cues if the two cues were at opposite locations (e.g., left and right; $33 \%$ of trials). We did not conduct similar analyses of the three-cue condition because the cued locations were always contiguous.

Mean RTs for two-cue trials were submitted to a 2 (age group; young adults and older adults) $\times 2$ (target location; cued and uncued) $\times 2$ (cue condition; adjacent and separated) mixed ANOVA. As found in the previous analysis, there were main effects of age group (older adults were slower than young adults), $F(1,38)=28.78, p<.0001$, and target location (cued RTs were slower than uncued RTs, reflecting IOR), $F(1,38)=70.34, p<.0001$. There was also an interaction between target location and cue condition, $F(1,54)=18.13, p<.001$. Examining IOR difference scores (cued RT minus uncued RT), effects were greater for adjacent cues $(22 \mathrm{~ms})$ than for separated cues $(8 \mathrm{~ms})$, although both effects were significantly greater than zero, both $t \mathrm{~s}(40)>7.90$, both $p \mathrm{~s}<.01$. The three-way interaction was not significant, $F<1$, indicating that the decline in IOR from adjacent to separated cues was similar for young adults and older adults. Mean RTs and IOR difference scores as a function of age group and cue adjacency are reported in Table 3.

Table 3 Mean reaction times (ms) for cue-adjacency analysis (2 cues) of Experiment 1

\begin{tabular}{llllll}
\hline & \multicolumn{2}{l}{ Older adults } & & \multicolumn{2}{l}{ Young adults } \\
\cline { 2 - 3 } \cline { 5 - 6 } & Adj & Sep & & Adj & Sep \\
\hline RT means & & & & \\
Cued & 413 & 407 & & 329 & 317 \\
Uncued & 390 & 399 & & 307 & 309 \\
IOR & $23^{*}$ & $8 \dagger$ & & $22^{*}$ & $8^{*}$ \\
RT SDs & & & & 51 \\
Cued & 47 & 44 & & 59 & 55 \\
Uncued & 50 & 52 & & 54 & 15 \\
IOR & 16 & 19 & 11 & 51 \\
\hline
\end{tabular}

Adj Adjacent cue trials, Sep separated cue trials, $R T$ reaction time, $I O R$ inhibition of return difference score (cued RT minus uncued RT), $S D$ standard deviation

*IOR scores were significantly greater than zero according to a onesample $t$ test, $p<.05$

$\dagger$ IOR scores were marginally greater than 0 according to a one-sample $t$ test, $.05<p<.10$ 
Vector averaging analyses We next analyzed whether the RT patterns were consistent with vector averaging and a gradient distribution of IOR. A gradient of inhibition would be reflected in reduced RTs with increasing cue-target distance. First, response times following single cues were submitted to a $2 \times 3$ mixed ANOVA with age group (young adults and older adults) and target location $\left(0,90\right.$, or $180^{\circ}$ away from the cue) as the between- and within-subjects factors, respectively. There was a significant effect of target location, $F(2,76)=45.56, p<.0001$, that did not interact with age group, $F(2,76)=1.83, p>.15$. Student Newman Keuls (SNK) post hoc tests indicated that, consistent with a gradient of inhibition, responses to targets presented at the same location as the cue (375 ms) were significantly slower than responses to targets presented $90^{\circ}$ away from the cue (360 ms), which in turn were significantly slower than responses to targets $180^{\circ}$ from (directly opposite) the cue (354 ms), $p \mathrm{~s}<.05$. Responses as a function of age group and angular distance for single cues are presented in the left panel of Fig. 2.

If vector averaging were occurring when orienting to multiple cues, then participants would respond to targets presented at the center location of three cues (the average direction of the three cues) more slowly than to targets presented at one of the outer two locations. In a 2 (age group; young and older adults) $\times 3$ (target location; centercued, side-cued, and uncued) mixed ANOVA, there was a main effect of target location, $F(2,76)=48.59, p<.0001$, that did not interact with age group, $F<1$. Consistent with predictions, SNK post hoc tests indicated that participants responded more slowly when the target was presented at the center of the three cued locations (366 ms) than when the target was presented at one of the two outer cued locations (361 ms), and participants responded to both types of cued targets significantly more slowly than to uncued targets
(345 ms), $p \mathrm{~s}<.05$. Reaction times as a function of age group and target location for the three-cue condition are presented in the right panel of Fig. 2.

\section{Discussion}

Both age groups demonstrated significant IOR effects, and the magnitude of IOR (as measured by comparing cued and uncued RTs, averaged across locations) did not diminish with age or with increasing cue number. As such, from a limited resources perspective, we could conclude that young and older participants had sufficient resources to simultaneously inhibit the return of attention to at least three locations. Like Abrams and Pratt (1996), we found evidence that relative cue location influenced IOR magnitude. In the two-cue condition, inhibition was greater for adjacent cues than for separated cues, consistent with the idea that young and older adults treated adjacent locations as an inhibited region. However, inhibitory effects were still found at separated locations, so IOR at multiple locations could not be completely explained with a regional interpretation.

Several pieces of evidence supported a vector/gradient interpretation of the data (Klein et al., 2005). First, with single cues, responses were slowest at the cued location and decreased as the angular distance between the target and the cue increased. Targets that were 90 and $180^{\circ}$ from the cue were both at uncued locations, but responses were significantly slower at the $90^{\circ}$ location than at the $180^{\circ}$ location. Importantly, a similar gradient pattern was found with three cues (see Fig. 2), suggesting that the gradient was distributed from the averaging orienting direction of multiple cues. When the target was presented at the center of the three cues (the average direction of the cues),
Fig. 2 Reaction times (in $\mathrm{ms}$ ) as a function of relative cue location in Experiment 1. RTs in the left panel are plotted as a function of the angular distance (in degrees) between the cue and the target for single-cue trials. RTs in the right panel are plotted as a function of target location (at the center-cued location, at a side-cued location, or at the uncued location) for three-cue trials

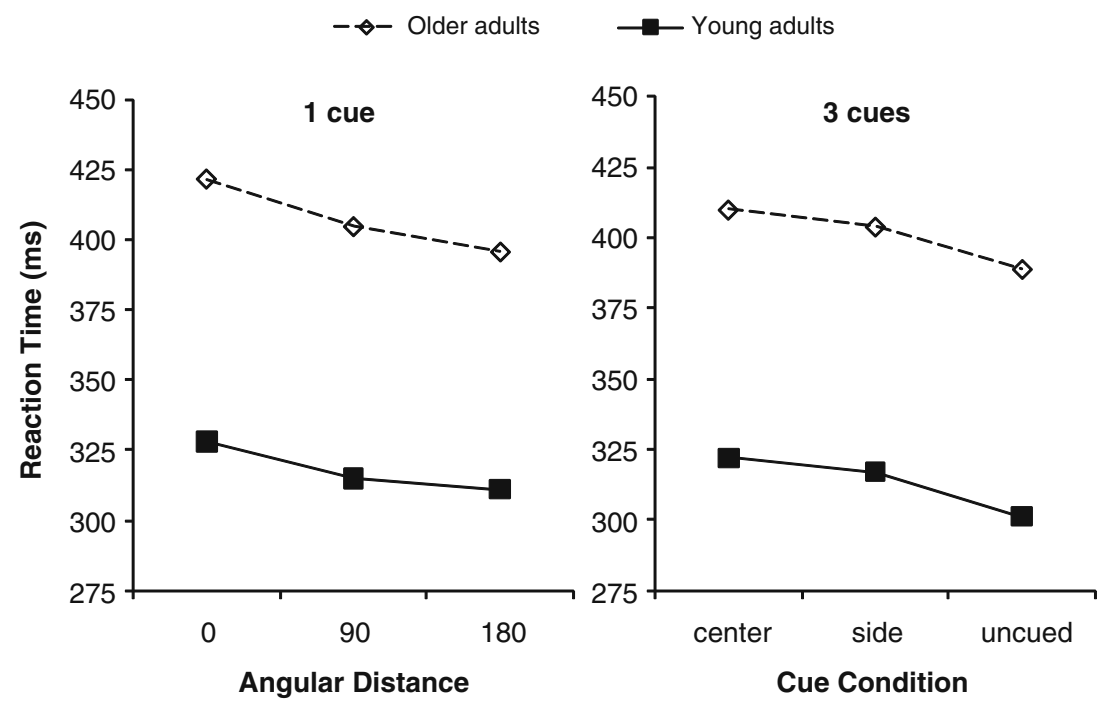


responses were slower than when the target was presented at one of the outer cued locations, and responses to the outer cued targets were slower than responses to targets at the uncued location (the furthest removed location from the average direction of the adjacent cues). We also found evidence for cue averaging with two cues. IOR was larger in magnitude for cues that were adjacently located (which would produce a relatively strong directional vector) than for cues that were at opposite sides of the array (which would produce a net vector at fixation). The finding of small but significant IOR for opposite cues cannot be easily explained by a vector interpretation, and is different from the Klein et al. (2005) finding of no significant IOR for zero net vector displays. This discrepancy will be discussed further in the General discussion. The gradient patterns did not vary with age group.

Together, there was empirical support for each of the three multiple-location IOR explanations. However, the data pattern was most consistent with a gradient of inhibition that spread from a point determined by the average direction of the cues. Older adults demonstrated the same inhibitory pattern as young adults in terms of the magnitude of inhibition and the gradient distribution of inhibition. As such, it appears that vector averaging processes and gradient distribution processes were not observably affected by age.

\section{Experiment 2}

From a limited resources perspective, the IOR pattern from Experiment 1 (no difference in the magnitude of IOR for one, two, and three cues, when cued and uncued RTs were averaged across array locations) suggested that young and older adults had sufficient resources to simultaneously inhibit returning attention to at least three locations. However, because visuospatial working memory resources are limited, it is possible that distribution beyond four or five locations would lead to diminished IOR, particularly for older adults. Another issue that arose from Experiment 1 was that, with a four-location array, there were limits to possible cue-target arrangements. Two cues were always adjacent to or opposite one another, and three cues were always adjacent. With more locations in the array, we could better test predictions from a regional and vector averaging perspective. Thus, in Experiment 2, we increased the number of potential target locations from four to eight, and we widened the range of cues to one, three, five, and seven cues.

Method

Participants Thirty-two young adults in the age range of 18-30 years (21 women, 11 men) and 32 older adults in the age range of 61-87 years (21 women, 11 men) were screened and tested in the same manner as described in Experiment 1. None of the participants from Experiment 2 participated in Experiment 1. Demographic and screening data for the included participants are reported in Table 1.

Materials, stimuli, and procedure We modified the display from Experiment 1 by adding boxes to the diagonal locations (top left, top right, bottom left, and bottom right) so that the resulting eight boxes of the array were arranged around the circumference of an imaginary circle. Sample cue arrays are presented in the top panels of Fig. 3. The boxes were the same distance from the fixation point $\left(7.15^{\circ}\right)$ and were the same size $\left(1.79^{\circ}\right.$ in width and height $)$ as the boxes in Experiment 1 .

After 28 practice trials, participants completed 896 test trials divided into 14 blocks of 64 trials. The trial sequence was identical to that of Experiment 1 except that one, three, five, or seven cues were presented prior to the target. Catch trials (trials in which the target was not presented) were presented on $14 \%$ of trials (128 trials total). The target's location was equally likely at the eight display positions, regardless of cue location. There were 24 cued trials and 168 uncued trials for the one-cue condition, 72 cued trials and 120 uncued trials for the three-cue condition, 120 cued trials and 72 uncued trials for the five-cue condition, and 168 cued trials and 24 uncued trials for the seven-cue condition.

\section{Results}

Limited resources analysis Mean RTs, IOR difference scores (cued RT minus uncued RT), and error rates for Experiment 2 are reported in Table 4. Errors, which were low overall (less than $2 \%$ for each age group), were

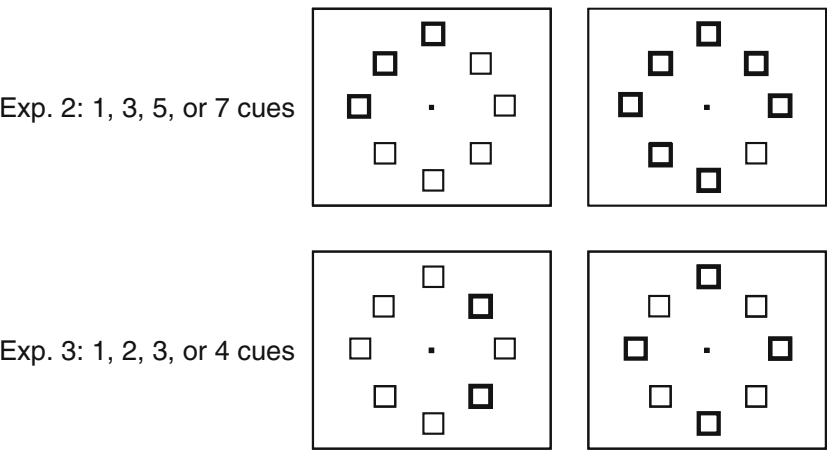

Fig. 3 Sample cue arrays for Experiment 2 (top row) and Experiment 3 (bottom row). The trial sequence and timing for Experiments 2 and 3 were the same as those used in Experiment 1. The arrays in the left panel represent cue arrangements with relatively strong vector amplitudes, and the arrays in the right panel represent cue arrangements with relatively weak vector amplitudes 
Table 4 Mean reaction times (ms) and error rates (\%) for Experiment 2
$R T$ reaction time, IOR inhibition of return difference score (cued RT minus uncued RT), $S D$ standard deviation

*IOR scores were significantly greater than zero according to a one-sample $t$ test, $p<.05$

\begin{tabular}{|c|c|c|c|c|c|c|c|c|}
\hline \multirow[b]{2}{*}{ Number of cues } & \multicolumn{4}{|c|}{ Older adults } & \multicolumn{4}{|c|}{ Young adults } \\
\hline & 1 & 3 & 5 & 7 & 1 & 3 & 5 & 7 \\
\hline \multicolumn{9}{|l|}{ RT means } \\
\hline Cued & 434 & 424 & 417 & 415 & 304 & 293 & 289 & 287 \\
\hline Uncued & 416 & 412 & 414 & 412 & 291 & 285 & 286 & 283 \\
\hline IOR & $18^{*}$ & $12^{*}$ & 3 & 3 & $13 *$ & $8^{*}$ & 3 & 4 \\
\hline \multicolumn{9}{|l|}{ RT SDs } \\
\hline Cued & 68 & 60 & 58 & 56 & 43 & 45 & 45 & 45 \\
\hline Uncued & 59 & 57 & 57 & 58 & 45 & 44 & 43 & 49 \\
\hline IOR & 26 & 11 & 11 & 15 & 16 & 12 & 10 & 16 \\
\hline \multicolumn{9}{|l|}{ Error rates } \\
\hline Cued & 0.4 & 0.3 & 0.3 & 0.3 & 0.5 & 0.6 & 0.6 & 0.7 \\
\hline Uncued & 0.4 & 0.2 & 0.3 & 0.4 & 0.7 & 0.5 & 0.8 & 0.5 \\
\hline
\end{tabular}

removed from the RT analysis. As in Experiment 1, RTs that were less than $150 \mathrm{~ms}$, more than $2,000 \mathrm{~ms}$, or more than 2.5 standard deviations away from a participant's condition mean were also eliminated. The means of the remaining RTs were submitted to a $2 \times 4 \times 2$ mixed ANOVA with age group (young adults and older adults) as the between-subjects factor and cue number $(1,3,5$, and 7 cues) and target location (cued and uncued) as the withinsubjects factors. All main effects were significant. Older adults were slower than young adults $(418$ and $290 \mathrm{~ms}$, respectively), $F(1,62)=99.52, p<.0001$, RT decreased as the number of cues increased $(361,354,351$, and $349 \mathrm{~ms}$ for $1,3,5$, and 7 cues, respectively), $F(3,186)=24.68, p<$ .0001 , and IOR was evidenced by slower RTs to targets at cued locations than to targets at uncued locations (358 and $350 \mathrm{~ms}$, respectively), $F(1,54)=52.91, p<.0001$. In addition, there was a significant two-way interaction between cue number and target location, $F(3,186)=11.47, p<$ .0001 , consistent with a change in IOR as the number of cues increased. IOR difference scores (cued RT minus uncued RT) were significantly greater for one cue than for three cues, which in turn were significantly greater than IOR scores for five or seven cues, as indicated by SNK post-hoc $t$ tests, $p \mathrm{~s}<.05$. There were no interactions involving age group, indicating that inhibitory patterns were similar for young and older adults.

Regional inhibition analysis To determine the influence of adjacent cues on IOR patterns, we examined data from the three-cue and five-cue conditions (cues in the seven-cue condition were always adjacent). In the three-cue condition, a trial was classified as adjacent if the three cued locations were all contiguous (e.g., top, top-right, and right; $14 \%$ of trials), and a trial was classified as separated if none of the three cued locations were adjacent (e.g., top, left, and bottom-right; $29 \%$ of trials). Trials in which two but not three of the cued boxes were adjacent (57\% of trials) were omitted from the analysis. In the five-cue condition, a trial was classified as adjacent if all five cued locations were adjacent (e.g., bottom, bottom-left, left, top-left, and top; 14\% of trials). In an eight-location display, it was impossible to have a five-cue condition in which all cues were separated (with uncued boxes on either side of a cued box), so trials were classified as separated if no more than two of the cued boxes were adjacent (resulting in two pairs of adjacent boxes; e.g., left, left-top, right-top, right, and bottom; $14 \%$ of trials). Fivecue trials in which three or four cued boxes were adjacent ( $72 \%$ of trials) were omitted from the analysis.

The RT data were submitted to a 2 (age group; young adults and older adults) $\times 2$ (cue number; three and five cues) $\times 2$ (target location; cued and uncued) $\times 2$ (cue condition; adjacent and separated) mixed ANOVA. There were main effects of age group (older adults were slower than young adults), $F(1,62)=102.37, p<.0001$, cue number (RTs were slower following three cues than following five cues), $F(1,62)=8.67, p<.01$, target location (reflecting IOR), $F(1,62)=40.06, p<.0001$, and cue condition (RTs were slower following adjacent than separated cues), $F(1,62)=5.62, p<.05$. Relevant to a regional inhibition interpretation, the only interaction that was significant was between target location and cue condition, $F(1,62)=17.16, p<.0001$, reflecting greater IOR effects for adjacent cues than for separated cues $(16$ and $6 \mathrm{~ms}$, respectively), although both effects were significantly greater than zero, both $t \mathrm{~s}(63)>6.0$, both $p \mathrm{~s}<.01$. There were no interactions involving age group, indicating that the decline in IOR from adjacent to separated cues was similar for young and older adults. Mean RTs and IOR difference scores for adjacent and separated trials are presented in Table 5.

Vector averaging analyses As in Experiment 1, we examined whether the distance between targets and cues 
Table 5 Mean reaction times ( $\mathrm{ms}$ ) for cue-adjacency analysis (3 and 5 cues) of Experiment 2

\begin{tabular}{llllll}
\hline & \multicolumn{2}{l}{ Older adults } & & & \multicolumn{2}{l}{ Young adults } \\
\cline { 2 - 3 } \cline { 5 - 6 } & Adj & Sep & & Adj & Sep \\
\hline RT means & & & & \\
Cued & 428 & 420 & & 298 & 291 \\
Uncued & 412 & 412 & & 283 & 286 \\
IOR & $16^{*}$ & $8^{*}$ & & $15^{*}$ & 5 \\
RT SDs & & & & 51 \\
Cued & 60 & 60 & & 51 & 49 \\
Uncued & 56 & 57 & 40 & 42 \\
IOR & 24 & 20 & 22 & 24 \\
\hline
\end{tabular}

Adj Adjacent cue trials, Sep separated cue trials, $R T$ reaction time, IOR inhibition of return difference score (cued RT minus uncued RT), $S D$ standard deviation

*IOR scores were significantly greater than zero according to a onesample $t$ test, $p<.05$

impacted reaction time. We used the analysis approach described by Klein and colleagues (2005), calculating the angular distance between the target and the average direction of the cues for all multiple-cue combinations. ${ }^{1}$ The calculated distances were binned into distances with means of $0,45,90,135$, and $180^{\circ}$. We limited the analysis to cue combinations in which the vector amplitude of the cues was greater than $25 \%$ of the distance from fixation to the imaginary circle on which the cues and targets were presented. This included all one-cue trials, $86 \%$ of three-cue trials, $43 \%$ of five-cue trials, and none of the seven-cue trials.

We submitted mean RTs for single- and multiple-cue trials to a $2 \times 2 \times 5$ mixed ANOVA with age group (young adults and older adults) as the between-subjects factor and number of cues (one or more than one) and cue-target angular distance $\left(0,45,90,135\right.$, and $\left.180^{\circ}\right)$ as the withinsubjects factors. All main effects were significant. Older adults were slower than young adults (418 and $290 \mathrm{~ms}$, respectively), $F(1,62)=97.28, p<.0001$, and responses were faster to multiple cues than to single cues (352 and $356 \mathrm{~ms}$, respectively), $F(1,62)=10.28, p<.01$. RTs varied as a function of angular distance, $F(4,248)=57.08, p<$ .0001 . SNK post-hoc tests revealed that RTs decreased with each binned increase in angular distance $(364,360,353,348$, and $344 \mathrm{~ms}$ for angular distances of $0,45,90,135$, and $180^{\circ}$, respectively), $p \mathrm{~s}<.05$.

There was an interaction between angular distance and number of cues, $F(4,248)=5.63, p<.001$, which we explored by examining RT differences between single and

\footnotetext{
${ }^{1}$ We extend our thanks to Ray Klein for providing the vector calculator and for recommending these analyses.
}

multiple cues at each angular distance. Differences were limited to small angular distances. Participants responded more slowly following single cues than following multiple cues at angular distances of $0^{\circ}, F(1,63)=14.14, p<.001$, and $45^{\circ}, F(1,63)=12.97, p<.001$, but not at greater angular distances, all $F_{\mathrm{s}}<1$. An alternative means of exploring the interaction was to calculate individual regression slopes representing the rate of change in $\mathrm{RT}$ as a function of cue-target distance. Submitted to a one-way cue number ANOVA, the slope for single cues $\left(-.140 \mathrm{~ms} /{ }^{\circ}\right)$ was significantly steeper than the slope for multiple cues $\left(-.087 \mathrm{~ms} /{ }^{\circ}\right), F(1,63)=13.94, p<.001$. None of the interactions involving age group were significant, all $F_{\mathrm{s}}<1$, consistent with an interpretation of a similar gradient pattern for young adults and older adults. RTs as a function of cue number and angular distance for young adults and older adults are presented in Fig. 4.

To determine whether inhibition was distributed based on the direction of the array's net vector, rather than parceled out to cued locations, we sorted multiple-cue data according to whether the target had been presented at a cued location or not. RTs were submitted to a 2 (age group; young and older adults) $\times 2$ (target location; cued and uncued) $\times 3$ (angular distance; 45, 90, and $135^{\circ}$ ) mixed ANOVA. The analysis was limited to angular distances of 45,90 , and $135^{\circ}$ because with an odd number of cues, only cued trials could create an angular distance of $0^{\circ}$, and only uncued trials could create an angular distance of $180^{\circ}$. All main effects were significant. Older adults were slower than young adults (417 and $289 \mathrm{~ms}$, respectively), $F(1,62)=$ $101.12, p<.0001$, responses were significantly slower at cued than uncued locations (356 and $351 \mathrm{~ms}$, respectively), $F(1,62)=16.80, p<.0001$, and participants responded more slowly to targets that were 45 and $90^{\circ}$ away from the net vector of the cues than to targets that were $135^{\circ}$ away $(357,354$, and $349 \mathrm{~ms}$, respectively), $F(2,124)=$ $9.11, p<.001$. None of the interactions were significant, all $F_{\mathrm{s}}<2.0$, all $p \mathrm{~s}>.20$.

A prediction based on a vector explanation of the data was that there would be minimal IOR on trials in which the net vector amplitude for multiple cues was close to zero (Klein et al., 2005). With an odd number of cues in an eight-location array, there were no true zero-amplitude arrays. However, we examined trials in which the net vector amplitude was less than $25 \%$ (14\% of three-cue trials, $57 \%$ of five-cue trials, and $100 \%$ of seven-cue trials). With RTs submitted to a 2 (age group; young and older adults) $\times 2$ (target location; cued and uncued) mixed ANOVA limited to low amplitude trials, there was again a main effect of age group (older adults were slower than young adults), $F(1,62)=100.46, p<.0001$, but there was not a significant effect of target location, $F(1,62)=1.39, p>$ .20. Responses were similar for cued and uncued trials 
Fig. 4 Reaction times (in $\mathrm{ms}$ ) as a function of cue number (one and more than one) and angular distance (in degrees) between the cue and the target for young adults and older adults for Experiment 2

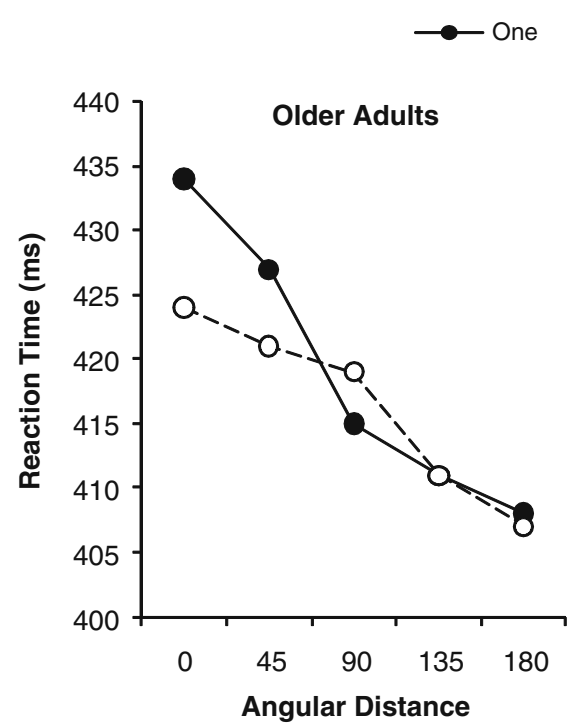

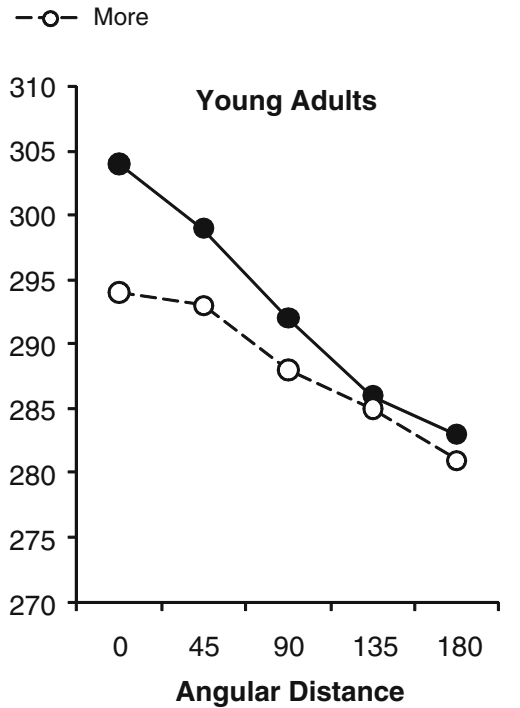

(352 and $351 \mathrm{~ms}$, respectively). Furthermore, the interaction with age was not significant, $F<1$. A companion analysis that assessed gradient patterns for low amplitude trials found that RTs did not vary as a function of binned cue-target angular distance (with RTs of 351, 353, 351, 351 , and $349 \mathrm{~ms}$ for angular distances of $0,45,90,135$, and $180^{\circ}$, respectively), $F(4,248)=1.85, p>.10$.

\section{Discussion}

When comparing response latencies averaged across all cued and uncued locations, we again observed that, from a limited resources perspective, IOR appeared to be associated with as many as three locations. In contrast, inhibitory effects were less than $5 \mathrm{~ms}$ in magnitude for five and seven cues, consistent with a limited pool of attentional resources that could be effectively distributed to only a certain number of locations (three or four). There was no evidence that this resource limit varied with age; young adults and older adults showed similar rates of diminishing IOR with increasing cue number. Because our task involved simultaneous rather than sequential cues, the decrease in IOR magnitude could not be attributed to temporal decay in IOR (Danziger et al., 1998).

In contrast to Experiment 1, in which IOR appeared to be constant in magnitude for one, two, and three cues, inhibitory effects in Experiment 2 declined from one to three cues and from three to five cues. The discrepancy could not be explained by a limited resources account. In the three-cue conditions of both Experiments 1 and 2, resources were spread across three locations, which should have resulted in similar IOR effects regardless of the number of array locations. But IOR for three cues was reduced in Experiment $2(10 \mathrm{~ms})$ relative to Experiment 1
(18 ms). A regional account of IOR would have predicted this decline. In both experiments, inhibitory effects were greater for adjacent cues than for separated cues, consistent with the interpretation that inhibition was largely limited to adjacent locations that could be considered a region. Because contiguous cues were more probable in Experiment 1 , and because the probability of contiguous cues increased with cue number $(67 \%$ for 2 cues, $100 \%$ for three cues), the regional effect of adjacent cues in Experiment 1 may have masked or countered the decline in IOR due to increasing cue number. With the eight-location display of Experiment 2, a small proportion of three-cue trials were contiguous (14\%), which may have unmasked the decline in IOR for multiple cues. However, as in Experiment 1, IOR was still significant for separated locations, so a regional explanation could not completely account for IOR observed across multiple locations.

Vector averaging of IOR could account for both the decline in IOR as cue number increased and the greater magnitude of IOR for three cues in Experiment 1 relative to Experiment 2. As the number of cues increased to seven, the cues were more likely to be spread around the circular array, and as such, the likelihood that the net vector of those cues was located close to fixation increased (with $14 \%$ of three-cue trials, $57 \%$ of five-cue trials, and $100 \%$ of sevencue trials having vector amplitudes less than $1.8^{\circ}$ of visual angle away from fixation). According to a vector explanation, if orienting remained close to fixation, then a gradient of inhibition would not be associated with a specific array location, so IOR would be smaller for displays with more cues. (Analyses did indicate that IOR effects were small in low amplitude arrays.) Vector averaging would have also predicted the between-experiment difference in the magnitude of three-cue IOR because in Experiment 1, with only four array locations, the three cues created a strong net 
vector (with the target being presented 0 or $90^{\circ}$ away from the net direction of the cues when it appeared at a cued location). In Experiment 2, the three cues could be spread among eight locations, and an individual cue would be more likely than in Experiment 1 to be removed from the average direction of orienting, resulting in less inhibition at that location. Thus, when comparing performance on cued and uncued trials as the indicator of IOR, inhibition would be greater for the array with fewer locations (Experiment 1).

Additional evidence in support of net vector averaging came from high amplitude (greater than $25 \%$ ) arrays. As the target increased in angular distance from the cue, there was a monotonic decrease in response times. This gradient pattern was observed with both single and multiple cues (but, importantly, not in low amplitude arrays). Evidence that vector averaging and a gradient distribution of IOR could not completely account for the observed inhibitory patterns came from the findings that (1) the rate of change in response times as a function of cue-target angular distance was greater for single cues than for multiple cues; and (2) for trials with multiple cues, in addition to the gradient pattern, there remained slowed responses to targets presented at locations stimulated by a cue.

The IOR patterns of Experiment 2 did not vary by age. Considering (1) IOR magnitude as a function of cue number, (2) the disparity in IOR for adjacent and separated locations, and (3) RTs as a function of cue-target angular distance for single and multiple cues, young adults and older adults demonstrated similar inhibitory patterns. Thus, there was no evidence for age differences in the spatial distribution of IOR.

\section{Experiment 3}

In Experiment 3, we examined IOR effects for one, two, three, and four cues within an eight-location array. The question of interest was, with a small number of cues, would we replicate the finding from Experiment 1 of unchanging IOR with increasing cue number? Or, because we reduced the frequency of neighboring cues by using eight rather than four array locations, would there be a decline in IOR with increasing cue number (like in Experiment 2)? We could also determine whether the vector patterns found by Klein et al. (2005) could be replicated in young adults and extended to older adults when using the number of cues and array locations that were used in their study.

Method

Participants Forty-four young adults in the age range of 18-24 years ( 25 women, 19 men), and 44 older adults in the age range of $60-82$ years ( 30 women, 14 men) were recruited and screened in the same manner as in the previous experiments, but none of the participants had participated in Experiments 1 or 2. Demographic and screening data for the included participants are presented in Table 1.

Materials, stimuli, and procedure The task was identical to that of Experiment 2 except that one, two, three, or four of the eight locations were cued (see sample arrays in Fig. 3, bottom panel). After 28 practice trials, participants completed 896 experimental trials divided into 14 blocks of 64 trials. Catch trials were presented on $14 \%$ of trials (128 total). The location of the target was equi-probable at the eight display locations. There were 24 cued trials and 168 uncued trials for the one-cue condition, 48 cued trials and 144 uncued trials for the two-cue condition, 72 cued trials and 120 uncued trials for the three-cue condition, and 96 cued trials and 96 uncued trials for the four-cue condition.

\section{Results}

Limited resources analysis Mean RTs, IOR difference scores (cued RT minus uncued RT), and error rates are presented in Table 6. Outlier RTs and error trials were removed in the same manner as described in Experiments 1 and 2. Mean RTs for the remaining trials were submitted to a $2 \times 4 \times 2$ mixed ANOVA with age group (young and older adults) as the between-subjects factor and cue number $(1,2,3$ and 4 cues) and target location (cued and uncued) as the withinsubjects factors. All main effects were significant. Older adults were slower than young adults $(399$ and $311 \mathrm{~ms}$, respectively), $F(1,86)=56.85, p<.0001$, RTs decreased as the number of cues increased $(364,354,353$, and $350 \mathrm{~ms}$ for $1,2,3$, and 4 cues, respectively), $F(3,258)=61.92, p<$ .0001 , and IOR was evidenced by slower RTs to targets at the cued locations than at the uncued locations (361 and $350 \mathrm{~ms}$, respectively), $F(1,86)=161.53, p<.0001$.

In addition to the main effects, there were two significant interactions: age group by target location, $F(1,86)=9.79$, $p<.01$, and cue number by target location, $F(3,258)=10.35$, $p<.0001$. Both young adults, $F(1,43)=71.07, p<.0001$, and older adults, $F(1,43)=92.61, p<.0001$, showed evidence of significant IOR effects (slower responses to cued than uncued targets); the age interaction was due to greater IOR effects for older adults than for young adults (13 and $8 \mathrm{~ms}$, respectively). For the cue number by target location interaction, SNK post-hoc $t$ tests indicated that IOR scores were significantly greater for one cue than for two, three, or four cues, $p \mathrm{~s}<.05$. For each age group tested independently, IOR effects were significantly greater than 0 for all four cues, all $t \mathrm{~s}(44)>2.5$, all $p \mathrm{~s}<.05$. 
Table 6 Mean reaction times (ms) and error rates (\%) for Experiment 3
$R T$ Reaction time, IOR inhibition of return difference score (cued RT minus uncued RT), SD standard deviation

*IOR scores were significantly greater than zero according to a one-sample $t$ test, $p<.05$

\begin{tabular}{|c|c|c|c|c|c|c|c|c|}
\hline \multirow[b]{2}{*}{ Number of cues } & \multicolumn{4}{|c|}{ Older adults } & \multicolumn{4}{|c|}{ Young adults } \\
\hline & 1 & 2 & 3 & 4 & 1 & 2 & 3 & 4 \\
\hline \multicolumn{9}{|l|}{ RT means } \\
\hline Cued & 419 & 404 & 402 & 399 & 327 & 312 & 313 & 309 \\
\hline Uncued & 398 & 393 & 392 & 389 & 314 & 307 & 305 & 303 \\
\hline IOR & $21 *$ & $11^{*}$ & $10^{*}$ & $10^{*}$ & $13 *$ & $5^{*}$ & $8^{*}$ & $6^{*}$ \\
\hline \multicolumn{9}{|l|}{ RT SDs } \\
\hline Cued & 68 & 67 & 70 & 65 & 43 & 38 & 42 & 41 \\
\hline Uncued & 69 & 67 & 66 & 65 & 40 & 40 & 42 & 41 \\
\hline IOR & 18 & 13 & 14 & 9 & 17 & 13 & 9 & 11 \\
\hline \multicolumn{9}{|l|}{ Error rates } \\
\hline Cued & 0.3 & 0.2 & 0.2 & 1.2 & 0.2 & 0.6 & 0.3 & 1.1 \\
\hline Uncued & 0.2 & 0.1 & 0.0 & 0.1 & 0.4 & 0.4 & 0.3 & 0.2 \\
\hline
\end{tabular}

Regional inhibition analysis We again examined IOR effects for adjacent and separated cues. In the two-cue condition, a trial was classified as adjacent if the two cues were contiguous (e.g., bottom, bottom-right; $28 \%$ of trials), and a trial was considered separated if the two cues were separated by at least one other cue (e.g., top right, top left; $72 \%$ of trials). In the three-cue condition, a trial was classified as adjacent if the three cued locations were all contiguous (e.g., top, top-right, and right; $14 \%$ of trials), and a trial was classified as separated if none of the three cued locations were adjacent (e.g., top, left, and bottom; $29 \%$ of trials). Trials in which two but not three of the cued boxes were adjacent ( $57 \%$ of trials) were omitted from the analysis. In the four-cue condition, a trial was classified as adjacent if all four cued locations were adjacent (e.g., bottom, bottom-left, left, and top-left; $11 \%$ of trials) and was classified as separated if none of the four cued locations were adjacent (e.g., top, bottom, left, right; 3\% of trials); other trials (85\%) were omitted from the analysis.

The data were submitted to a 2 (age group; young adults and older adults) $\times 3$ (cue number; 2,3 , and 4 cues) $\times 2$ (target location; cued and uncued) $\times 2$ (cue location; adjacent and separated) mixed ANOVA. There were main effects of age group (older adults were slower than young adults), $F(1,86)=56.42, p<.0001$, cue number (RTs decreased as cue number increased), $F(2,172)=5.14$, $p<.01$, and target location (reflecting IOR), $F(1,86)=$ $43.89, p<.0001$. Two-way interactions were found between age group and target location, $F(1,86)=4.75$, $p<.05$, and between target location and cue location, $F(1,62)=6.64, p<.05$. As in the previous analysis, the interaction between age group and target location was due to larger IOR scores for older adults than for young adults (14 and $7 \mathrm{~ms}$, respectively). The interaction between target location and cue location was due to greater IOR scores for adjacent cues than for separated cues (14 and $7 \mathrm{~ms}$, respectively), replicating the patterns from Experiments 1 and 2. Inhibitory effects were significant for both adjacent and separated cues, both $t \mathrm{~s}(87)>2.5$, both $p \mathrm{~s}<.01$. RTs and IOR scores for adjacent and separated locations are reported in Table 7.

Vector averaging analyses We examined whether, beyond a target being cued or uncued, the distance between targets and cues impacted response time. As in Experiment 2, we calculated the angular distance between the target and the average direction of cues. These distances were sorted into bins with mean distances of $0,45,90,135$, and $180^{\circ}$. For cue combinations in which the vector amplitude (directional strength) was greater than $25 \%$ (which was true for $100 \%$ of one-cue trials, $86 \%$ of two-cue trials, $85 \%$ of three-cue trials, and $69 \%$ of four-cue trials), mean RTs were

Table 7 Mean reaction times (ms) for the cue-adjacency analysis (2, 3, and 4 cues) of Experiment 3

\begin{tabular}{llllll}
\hline & \multicolumn{2}{l}{ Older adults } & & \multicolumn{2}{l}{ Young adults } \\
\cline { 2 - 3 } \cline { 5 - 6 } & Adj & Sep & & Adj & Sep \\
\hline RT means & & & & 310 \\
Cued & 408 & 400 & & 314 & 306 \\
Uncued & 390 & 390 & & 304 & 30 \\
IOR & $18^{*}$ & $10^{*}$ & & $10^{*}$ & 4 \\
RT SDs & & & & 42 \\
Cued & 72 & 70 & & 42 & 48 \\
Uncued & 67 & 70 & 44 & 50 \\
IOR & 25 & 37 & 27 & 44 \\
\hline
\end{tabular}

Adj Adjacent cue trials, Sep separated cue trials, $R T$ reaction time, IOR inhibition of return difference score (cued RT minus uncued RT), $S D$ standard deviation

*IOR scores were significantly greater than zero according to a onesample $t$ test, $p<.05$ 
submitted to a 2 (age group; young adults and older adults) $\times 2$ (number of cues; one or more than one) $\times 5$ (cue-target angular distance; $0,45,90,135$, and $180^{\circ}$ ) mixed ANOVA. All main effects were significant. Older adults were slower than young adults (398 and $312 \mathrm{~ms}$, respectively), $F(1,86)=53.73, p<.0001$, responses were faster following multiple cues than following single cues $(352$ and $359 \mathrm{~ms}$, respectively), $F(1,86)=52.95, p<.0001$, and RTs decreased with increasing angular distance $(367,362,353,348$, and $346 \mathrm{~ms}$ for angular distances of $0,45,90,135$, and $180^{\circ}$, respectively), $F(4,344)=103.06, p<.0001$. SNK post hoc $t$ tests confirmed that each change in angular distance was accompanied by a significant change in RT, except from 135 to $180^{\circ}, p \mathrm{~s}<.05$.

In addition to the main effects, there were two significant interactions: age group by angular distance, $F(4,344)=$ $4.57, p<.01$, and number of cues by angular distance, $F(4,344)=4.94, p<.001$. Exploring the interaction involving age, both age groups showed distance effects, both $F \mathrm{~s}>35.00$, both $p \mathrm{~s}<.0001$. Collapsing across the number of cues, we calculated individual regression slopes representing the rate of RT change as a function of angular distance. Based on a one-way ANOVA, slopes were steeper for older adults than for young adults $\left(-.139\right.$ and $-.095 \mathrm{~ms} /{ }^{\circ}$, respectively), $F(1,86)=7.58, p<.01$, indicating a faster decline in RT as angular distance increased for the older group. To explore the cue number interaction, we examined cue number effects at each of the five binned angular distances (including participants from both age groups). Responses were consistently slower following single cues than following multiple cues, all $F \mathrm{~s}>5.0$, all $p \mathrm{~s}<.05$. We then calculated RT regression slopes for single and multiple cues and found that slopes were significantly steeper for single cues than for multiple cues $\left(-.141\right.$ and $-.104 \mathrm{~ms} /{ }^{\circ}$, respectively), $F(1,87)=10.23, p<.01$. Figure 5 depicts
RTs as a function of angular distance and cue number for each age group.

We sorted multiple-cue data according to whether the target had been presented at a cued location or not. If inhibition was distributed as a gradient based on the net direction of the cues, then responses should have been similar to cued and uncued locations. Mean RTs for multiple-cue trials were submitted to a 2 (age group; young and older adults) $\times 2$ (target location; cued and uncued) $\times 5$ (angular distance; $0,45,90,135$, and $\left.180^{\circ}\right)$ mixed ANOVA. All main effects were significant. Older adults were slower than young adults (397 and $309 \mathrm{~ms}$, respectively), $F(1,86)=57.68, p<.0001$, responses were slower in the cued than in the uncued condition $(354$ and $351 \mathrm{~ms}$, respectively), $F(1,86)=7.34, p<.01$, and RTs decreased as angular distance increased $(361,358,350,348$, and $346 \mathrm{~ms}$ for distances of $0,45,90,135$, and $180^{\circ}$, respectively), $F(4,344)=35.47, p<.0001$. In addition to the main effects, there was an interaction between target location and angular distance, $F(4,344)=3.36, p<.05$. There was not a cuing effect (slower RT to cued than uncued targets) at $0^{\circ}(-3 \mathrm{~ms})$ or at $90^{\circ}(1 \mathrm{~ms})$, both $p \mathrm{~s}>.10$, but cuing effects were significant at $45^{\circ}(5 \mathrm{~ms}), F(1,87)=8.89, p<.01$, and at $135^{\circ}(6 \mathrm{~ms})$, $F(1,87)=8.10, p<.01$, and they were marginally significant at $180^{\circ}(5 \mathrm{~ms}), F(1,87)=2.94, .05<p<.10$.

With an even number of cues (two and four cues) in an eight-location array, there were trials with true zeroamplitude net vectors (e.g., two cues presented in the top and bottom locations, or four cues presented at the top, left, bottom, and right locations). A vector averaging account would predict no IOR for such trials because the average orienting direction of the cues would be at fixation. With RTs submitted to a 2 (age group; young and older adults) $\times$ 2 (target location; cued and uncued) mixed ANOVA limited to zero amplitude trials, there were main effects of age group, $F(1,86)=55.43, p<.0001$, and target location,
Fig. 5 Reaction times (in $\mathrm{ms}$ ) as a function of cue number (one, more than one) and cuetarget angular distance (in degrees) for young and older adults for Experiment 3
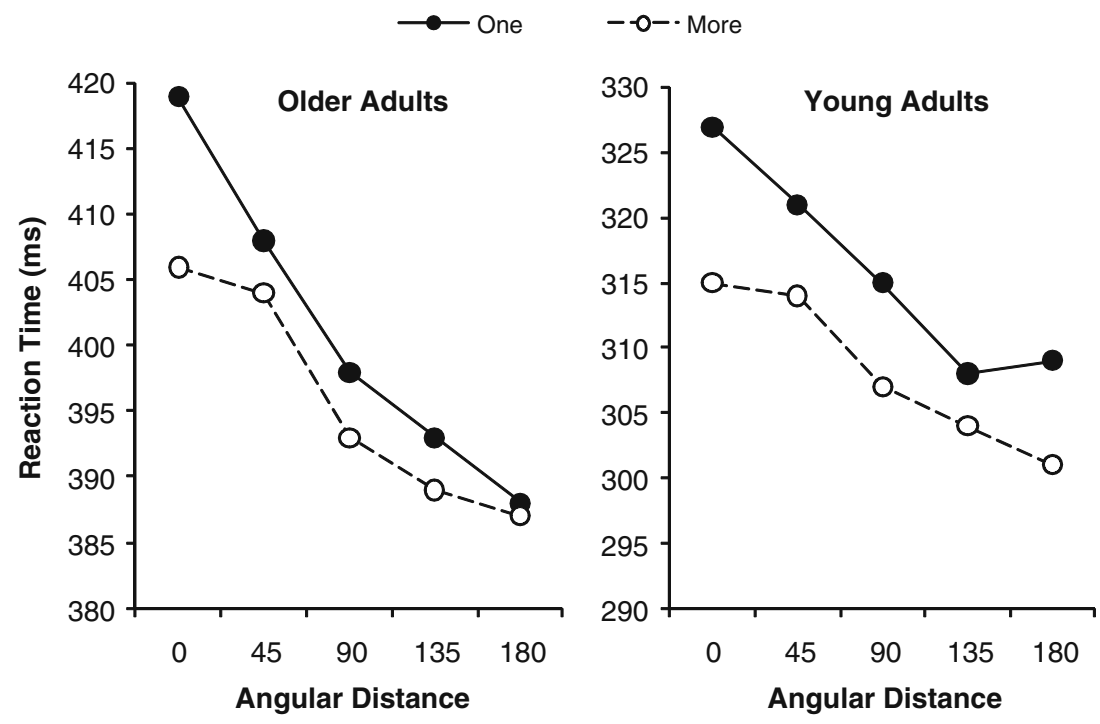
$F(1,86)=4.47, p<.05$. Older adults were slower than young adults (394 and $308 \mathrm{~ms}$, respectively), and responses were slower in the cued than in the uncued condition (353 and $348 \mathrm{~ms}$, respectively). In addition, the age group by target location interaction was significant, $F(1,86)=5.39, p<.05$. The cue effect (slower RTs in the cued than in the uncued condition) was significant for older adults (10 ms), $F(1,43)=11.93, p<.01$, but not for young adults $(0 \mathrm{~ms})$, $F<1$. To make comparisons with Experiment 2, we repeated the above analysis but included all trials with vector amplitudes under $25 \%$ (14\% of two-cue trials, $15 \%$ of three-cue trials, and $31 \%$ of four-cue trials). There were again main effects of age group, $F(1,86)=57.19, p<.0001$, and target location, $F(1,86)=21.81, p<.0001$, but the interaction between age group and target location was now only marginally significant, $F(1,86)=2.95, p<.10$. The cue effect was significant for both older adults $(8 \mathrm{~ms})$, $F(1,43)=20.55, p<.0001$, and for young adults (4 ms), $F(1,43)=4.33, p<.05$. A companion analysis that assessed gradient patterns for low amplitude trials found that RTs did not vary as a function of binned cue-target angular distance (with RTs of 351, 353, 350, 351, and $354 \mathrm{~ms}$ for angular distances of $0,45,90,135$, and $180^{\circ}$, respectively), $F(4,344)<1$.

\section{Discussion}

From a limited resources perspective, it appeared that young and older adults maintained IOR at up to four locations simultaneously, and the magnitude of IOR declined when distributed among multiple locations. We found no evidence for an age-related decline in inhibitory resources; in fact, IOR was greater in magnitude for older adults than for young adults, and the change in IOR with cue number did not vary with age.

We again found IOR patterns that were consistent with regional distribution of inhibition. IOR effects were greater when cues were adjacent to one another than when they were separated. In addition, although not compared statistically, IOR effects for two and three cues in Experiment 3 ( 8 and $9 \mathrm{~ms}$, respectively) were smaller than those in Experiment 1 (17 and $17 \mathrm{~ms}$, respectively). This cross-experiment reduction in IOR for multiple cues may be explained by a reduced likelihood of adjacent cues in Experiment 3 . Compared to $28 \%$ of two-cue trials and $14 \%$ of three-cue trials being adjacent in the present eightlocation display, $67 \%$ of two-cue trials and $100 \%$ of threecue trials were adjacent in Experiment 1's four-location display. Greater opportunity for regional effects may have bolstered IOR in Experiment 1, but as in the earlier experiments, IOR in the present experiment was significant even when cued locations were separated in space, so regional effects could not completely account for IOR patterns.

Vector averaging could also account for the influence of relative cue location. When multiple cues were distributed among eight locations rather than four, the net amplitude of the cues was more often close to fixation. Thus, the low amplitude trials would reduce IOR for multiple-cue trials relative to single-cue trials. And adjacent cues would be associated with a stronger net vector (and thus greater IOR) than separated or distributed cues. Vector averaging uniquely predicted angular distance effects. Each binned increase in angular distance was accompanied by a significant decrease in RT, except from 135 to $180^{\circ}$. The distance effect was observed for the average direction of multiple cues as well as for single cues, so the RT pattern was consistent with averaging of cues. Consistent with the findings of Experiment 2, responses were slower following single cues than following multiple cues, and the slope of the distance effect was steeper for single cues. Responses were particularly slowed when a target was presented close to a single cue, suggesting that single cues drew a stronger orienting response than multiple cues. Evidence that IOR was tagged to individual cued locations came from comparing cued and uncued trials in multiple-cue arrays. Although it was a small cuing effect ( $3 \mathrm{~ms})$, responses were slower when the target was presented at cued than uncued locations, indicating that responses were not completely driven by the average direction of the cues. In addition, when examining responses on zero amplitude and low amplitude trials (i.e., when the center of gravity of multiple cues was at or close to fixation), participants were slower on cued than uncued trials, again suggesting some role for local stimulation from cued locations.

The response patterns of both young and older adults were consistent with vector averaging, suggesting that these averaging processes were not impacted by age. The slope of the gradient was steeper for older adults, with notable inhibitory slowing for older adults at short cue-target distances. In addition, local stimulation effects may have been greater for older adults than for young adults, as reflected in greater cuing effects on zero and low amplitude trials for older adults, when inhibition would not be expected.

\section{General discussion}

The present study investigated the means by which young and older adults distributed inhibition to multiple locations. We observed IOR following as many as four simultaneous cues, replicating previous findings with young adults (Klein et al., 2005; Wright \& Richard, 1996), and extending this finding to older adults. We compared three explanations for 
the IOR patterns: allocation of limited inhibitory resources to stimulated locations, regional spread of inhibition to contiguous locations, and a gradient distribution of inhibition based on vector averaging of multiple cues. We found that vector averaging best accounted for the observed inhibitory patterns.

Limited inhibitory resources Considering the inhibitory pattern across the three experiments, IOR effects (as traditionally measured) were strongest for a single cue, diminished in strength for two, three, and four cues, and were minimal in size for five and seven cues. From a visuospatial working memory interpretation of IOR (Castel, Pratt, \& Craik, 2003; Pratt \& Chasteen, 2007), one could conclude that resources were sufficient to tag and inhibit four, but not more, attended locations in working memory. There was no evidence of age differences in this form of working memory. In fact, in Experiment 3, older adults' inhibitory effects were significantly greater than those of young adults. Consistent with the present findings, Pratt and Chasteen (2007) found similar IOR patterns for young adults and older adults on a sequential cuing task (with five locations inhibited). They concluded that the form of visuospatial working memory used in deployment of IOR is resistant to age-related decline because it is closely linked with the saccadic oculomotor system, which is relatively spared from aging effects.

Regional inhibition Inhibitory patterns varied based on the relative location of cues. Inhibitory effects were greater, for both young and older adults, when cued locations were contiguous rather than separated in space. In addition, when multiple cues were adjacently located, there was little difference in the magnitude of IOR relative to a single cued location, consistent with the idea that adjacently-cued locations were treated as a single inhibited region (Abrams \& Pratt, 1996). For example, in Experiment 2, IOR effects for adjacent cues (three and five cues) were 15 and $16 \mathrm{~ms}$ for young and older adults, respectively, and the corresponding IOR effects for single cues were 14 and $18 \mathrm{~ms}$. However, IOR to multiple-cued locations could not be entirely explained with a regional explanation. When limiting the analyses to trials in which the cued locations were separated by at least one uncued location, IOR effects, although smaller than those to contiguous cues, were still significantly greater than zero.

The question is whether there is an explanation for the residual IOR that is consistent with a regional account of IOR. One could imagine that when multiple separated cues were presented, instead of distributing attention to the cued locations, observers oriented to a single cue. Attention to a single location would result in inhibition at that location but not at the other cued locations. Thus, averaged across cued trials (those in which the target was presented at the attended location, and those in which the target was presented at an unattended cued location), one would expect to see inhibition of a reduced magnitude relative to single or adjacent trials (which is what we observed), even if IOR was not associated with multiple locations. However, the magnitude of IOR for separated cues did not vary significantly based on the number of cued locations (see Experiments 2 and 3). One would expect that attending to one of two cued locations would result in IOR on approximately half of the cued trials, whereas attending to one of four cued locations would result in IOR on approximately one-quarter of trials. This did not appear to be the case; the interaction between target location (cued and uncued) and cue location (adjacent or separated) was not qualified by cue number. Thus, the evidence argues against IOR being limited to single cued locations or regions under multiple cue conditions.

Vector averaging and gradient distribution of inhibition Although the present IOR patterns could be interpreted from the viewpoints of limited inhibitory resources and regional distribution of inhibition, vector averaging of multiple locations (Klein et al., 2005) best accounted for the full range of observed IOR effects. A unique prediction based on vector averaging was that a gradient of inhibition would be distributed from the net direction of multiple cues. In all three experiments, response times following a single cue were slowest to targets at the cued location and gradually decreased as targets increased in distance from the cue, consistent with inhibition that radiated from the initial point of orientation (Bennett \& Pratt, 2001; Maylor \& Hockey, 1985; Pratt et al., 1999). Replicating Klein et al., we found that multiple cues initiated a similar directional gradient centered on the net vector of cues. Thus, when the center of gravity of multiple cues was at least $1.8^{\circ}$ from fixation and was capable of initiating an orienting response, inhibition could be generated for locations that had not even been cued. The two alternative explanations (limited inhibitory resources and regional distribution of IOR) could not account for this gradient pattern.

Additional support for vector averaging of multiple cues came from analyses of zero and low amplitude arrays (in which the center of gravity of the cues fell at or close to fixation). Klein and colleagues (2005) found that response times in zero amplitude arrays did not differ significantly between cued and uncued trials. In Experiments 2 and 3, we found minimal cuing effects $(0-4 \mathrm{~ms})$ and no gradient effects for young adults in zero and low amplitude arrays. In Experiment 1, however, we observed IOR of approximately $8 \mathrm{~ms}$ (for both young and older adults) when two cues were presented at opposite locations (with a center of gravity at fixation). Additionally, zero- and low-amplitude 
IOR for older adults was $8-10 \mathrm{~ms}$ in Experiment 3 . Together, the evidence from low-amplitude arrays indicates that the net vector elicited by the cues more so than the individual cues guided inhibition, although there was some contribution by the individual cues in generating inhibition, particularly for older adults.

When the target was at or near the cued location or net direction of cues, there was a small advantage to a single stimulated location in inhibiting the return of attention to that location. Response times were slower following single cues than following multiple cues (a 4-ms effect in Experiment 2, a 7-ms effect in Experiment 3), particularly at smaller angular distances $\left(0\right.$ and $\left.45^{\circ}\right)$. Note that although Klein and colleagues (2005) found a non-significant difference between single and multiple cues, their effect was similar in magnitude (approximately $5 \mathrm{~ms}$ ) to that found in the present study. The faster responses for multiple-cue trials may be explained by the fact that the center of gravity of multiple cues (the location at which inhibition was strongest) often fell short of the imaginary circle on which targets were presented, whereas a target following a single cue was presented directly at the cued (inhibited) location. The discrepancy in response latency between single-cued and multiple-cued trials may also be due to local stimulation effects, with an inhibitory advantage when attention is directed toward a single cued location. Additional evidence for small stimulation effects specific to the cued locations (rather than the net vector of cues) came from comparing cued and uncued responses to multiple-cue trials. If inhibition were distributed based on the net direction of cues rather than distributed to individual locations, then responses to targets at cued locations should not have differed from responses to targets at uncued locations. However, for trials with a substantial net vector, responses were slightly but significantly slower at cued than at uncued locations (by $5 \mathrm{~ms}$ in Experiment 2, and by $3 \mathrm{~ms}$ in Experiment 3), consistent with local stimulation. Note that the cuing effect for multiple-cue trials from the Klein et al. study, although not statistically significant, was similar in magnitude (3-4 ms).

The small local stimulation effects observed in the present study may have been caused, at least in part, by eye movements. We did not track eye movements or remove trials in which eye movements were made. A relatively long cue-target $\mathrm{SOA}(1,000 \mathrm{~ms})$ provided participants an opportunity to saccade to one or more cued locations before the target was presented. Klein and colleagues (2005) found that eye movements occurred on $30 \%$ of trials. In our study, location placeholders (boxes) that remained on the screen throughout a trial (placeholders were not used by Klein et al.) may have provided a spatial marker for a cue's location, to which participants could overtly orient even after the cue had been removed. Local stimulation effects may have been small because the effect was limited to those trials and locations to which eye movements occurred. However, it is worth noting that Klein found stimulation effects that were similar in size (although not statistically significant) even after removing trials with eye movements from the analyses.

Considered together, how do the present results inform the question of whether IOR is caused by covert orienting to the average direction of multiple cues or by local stimulation from individual cued locations? The clearly demonstrated gradient pattern for multiple cues that largely mimicked that found for single cues is strong evidence in support of a covert orienting account of IOR. In contrast, the small cuing effects associated with zero net vector trials and multiple-cue trials, and the small increase in response times associated with single cues as opposed to multiple cues, suggests that there is a contribution from local stimulation effects. However, the small magnitude of the effects suggests that local stimulation is not the primary driver of the IOR patterns, but rather modifies the primary IOR patterns driven by covert orienting.

From a vector averaging standpoint, age did not alter the processes by which the average direction of cues was computed. In Experiments 1 and 2, inhibitory gradient patterns for single and multiple cues were the same for young and older adults. In Experiment 3, older adults' gradient patterns were steeper than those for young adults, suggesting that for older adults, inhibitory effects were particularly strong at short cue-target distances (consistent with greater IOR effects for older adults in the experiment overall), or that older adults did not spread IOR to the greater cue-target distances as effectively. As found with young adults, there were indicators of local stimulation as well as covert orienting in older adults' inhibitory effects for multiple cue trials. In Experiment 3, older adults demonstrated greater cuing effects than did young adults for zero and low amplitude arrays, perhaps due to a greater number of saccades to cued locations.

To recap the age-relevant findings of the present study, regardless of the inhibitory viewpoint used to interpret the findings, minimal age differences were found in the spatial distribution of IOR. Young and older adults showed similar decline in IOR as a function of the number of simultaneous cues, indicating no age-related depletion in inhibitory resources. Both young and older adults showed a similar advantage for inhibition of adjacent versus separated locations, thus demonstrating similar regional distribution of IOR. Finally, gradient patterns varied little with age, leading to the conclusion that vector averaging of multiple simultaneously-cued locations was not vulnerable to age effects. Thus, although aspects of IOR functioning, such as temporal properties, do change with age (Castel, Chasteen, Scialfa, \& Pratt, 2003; Langley, Fuentes, Vivas, \& Saville, 
2007; McCrae \& Abrams, 2001), the spatial distribution of IOR does not appear to be one of them.

To conclude, results from the present study established that IOR could be observed following two, three, and four simultaneous cues, but less reliably following five or more cues. The pattern of findings was most consistent with vector averaging of cued locations. Age had little impact on the spatial distribution of IOR, consistent with relative preservation of this form of inhibitory allocation later in life.

Acknowledgments This work was supported by National Science Foundation Grant 01322899 and by Centers of Biomedical Research Excellence (COBRE) Grant P20 RR020151 from the National Center for Research Resources (NCRR), a component of the National Institutes of Health (NIH). The contents of this report are solely the responsibility of the authors and do not necessarily reflect the official views of the NSF, NIH, or NCRR. We are grateful to Atiana Stark, Jaryn Allen, Laura Klubben, Savannah Kraft, Shelly Manger, Marie Schaaf, Melissa Tarasenko, Lindsay Anderson, Heather Wadeson, Heather Joyce, Tanya Peterson, RaeAnn Levang, Nicole Kiewel, Amanda Benz, Emily Wegner, Carrie Spillers, Erin Beske, Alex McCrosky, Allison Deraney, Sara Wyman, Sabrina Thompson, and Sarah Nelson for their assistance in collecting data.

\section{References}

Abrams, R. A., \& Pratt, J. (1996). Spatially diffuse inhibition affects multiple locations: A reply to Tipper, Weaver, and Watson (1996). Journal of Experimental Psychology: Human Perception and Performance, 22, 1294-1298. doi:10.1037//0096-1523.22. 5.1294

Bennett, P. J., \& Pratt, J. (2001). The spatial distribution of inhibition of return. Psychological Science, 12, 76-80. doi:10.1111/14679280.00313

Birmingham, E., \& Pratt, J. (2005). Examining inhibition of return with onset and offset cues in the multiple-cuing paradigm. Acta Psychologica, 118, 101-121. doi:10.1016/j.actpsy.2004.10.005

Castel, A. D., Chasteen, A. L., Scialfa, C. T., \& Pratt, J. (2003). Adult age differences in the time course of inhibition of return. Journal of Gerontology: Psychological Sciences, 58B, P256-P259.

Castel, A. D., Pratt, J., \& Craik, F. I. M. (2003). The role of spatial working memory in inhibition of return: Evidence from divided attention tasks. Perception \& Psychophysics, 65, 970-981.

Christensen, K. J., Moye, J., Armson, R. R., \& Kern, T. M. (1992). Health screening and random recruitment for cognitive aging research. Psychology and Aging, 7, 204-208. doi:10.1037//08827974.7.2.204

D'Aloisio, A., \& Klein, R. M. (1990). Aging and the deployment of visual attention. In J. T. Enns (Ed.), The development of attention: Research and theory (pp. 447-466). Oxford: NorthHolland. doi:10.1016/S0166-4115(08)60470-7

Danziger, S., Kingstone, A., \& Snyder, J. J. (1998). Inhibition of return to successively stimulated locations in a sequential visual search paradigm. Journal of Experimental Psychology: Human Perception and Performance, 24, 1467-1475. doi:10.1037// 0096-1523.24.5.1467

Dodd, M. D., Castel, A. D., \& Pratt, J. (2003). Inhibition of return with rapid serial shifts of attention: Implications for memory and visual search. Perception \& Psychophysics, 65, 1126-1135.
Faust, M. E., \& Balota, D. A. (1997). Inhibition of return and visuospatial attention in healthy older adults and individuals with dementia of the Alzheimer type. Neuropsychology, 11, 13-29. doi:10.1037//0894-4105.11.1.13

Folstein, M. F., Folstein, S. E., \& McHugh, P. R. (1975). Mini-mental state: A practical method for grading the cognitive state of the patient for the clinician. Journal of Psychiatric Research, 12, 189-198. doi:10.1016/0022-3956(75)90026-6

Foster, J. K., Behrmann, M., \& Stuss, D. T. (1995). Aging and visual search: Generalized cognitive slowing or selective deficit in attention? Aging and Cognition, 2, 279-299. doi:10.1080/ 13825589508256604

Gottlob, L. R. (2006). Age-related deficits in guided search using cues. Psychology and Aging, 21, 526-534. doi:10.1037/08827974.21.3.526

Greenwood, P. M., \& Parasuraman, R. (2004). The scaling of spatial attention in visual search and its modification in healthy aging. Perception \& Psychophysics, 66, 3-22.

Hartley, A. A., \& Kieley, J. M. (1995). Adult age differences in the inhibition of return of visual attention. Psychology and Aging, 10, 670-683. doi:10.1037//0882-7974.10.4.670

Humphrey, D. G., \& Kramer, A. F. (1997). Age differences in visual search for feature, conjunction, and triple-conjunction targets. Psychology and Aging, 12, 704-717. doi:10.1037// 0882-7974.12.4.704

Klein, R. M. (1988). Inhibitory tagging system facilitates visual search. Nature, 334, 430-431. doi:10.1038/334430a0

Klein, R. M. (2000). Inhibition of return. Trends in Cognitive Sciences, 4, 138-147. doi:10.1016/S1364-6613(00)01452-2

Klein, R. M. (2005). On the role of endogenous orienting in the inhibitory aftermath of exogenous orienting. In U. Mayr, E. Awh, \& S. W. Keele (Eds.), Developing individuality in the human brain: A tribute to Michael I. Posner (pp. 45-64). Washington, DC: American Psychological Association. doi:10.1037/11108-003

Klein, R. M., Christie, J., \& Morris, E. P. (2005). Vector averaging of inhibition of return. Psychonomic Bulletin \& Review, 12, 295-300.

Langley, L. K., Fuentes, L. J., Vivas, A. B., \& Saville, A. L. (2007). Aging and temporal patterns of inhibition of return. Journal of Gerontology: Psychological Sciences, 62B, P71-P77.

Langley, L. K., Vivas, A. B., Fuentes, L. J., \& Bagne, A. G. (2005). Differential age effects on attention-based inhibition: Inhibitory tagging and inhibition of return. Psychology and Aging, 20, 356360. doi:10.1037/0882-7974.20.2.356

Maylor, E. A. (1985). Facilitatory and inhibitory components of orienting in visual space. In M. I. Posner \& O. S. M. Marin (Eds.), Attention and performance XI (pp. 189-204). Hillsdale, NJ: Erlbaum.

Maylor, E. A., \& Hockey, R. (1985). Inhibitory component of externally controlled covert orienting in visual space. Journal of Experimental Psychology: Human Perception and Performance, 11, 777-787. doi:10.1037//0096-1523.11.6.777

McCrae, C. S., \& Abrams, R. A. (2001). Age-related differences in object- and location-based inhibition of return of attention. Psychology and Aging, 16, 437-449. doi:10.1037//08827974.16.3.437

Posner, M. I., \& Cohen, Y. (1984). Components of visual orienting. In H. Bouma \& D. G. Bowhuis (Eds.), Attention and performance $X$ (pp. 531-556). Hillsdale, NJ: Erlbaum.

Pratt, J., \& Chasteen, A. L. (2007). Examining inhibition of return with multiple sequential cues in younger and older adults. Psychology and Aging, 22, 404-409. doi:10.1037/0882-7974.22.2.404

Pratt, J., Spalek, T. M., \& Bradshaw, F. (1999). The time to detect targets at inhibited and noninhibited locations: Preliminary evidence for attentional momentum. Journal of Experimental Psychology: Human Perception and Performance, 25, 730-746. doi:10.1037//0096-1523.25.3.730 
Rösler, A., Mapstone, M., Hays-Wicklund, A., Gitelman, D. R., \& Weintraub, S. (2005). The "zoom lens" of focal attention in visual search: Changes in aging and Alzheimer's disease. Cortex, 41, 512-519. doi:10.1016/S0010-9452(08)70191-6

Snyder, J. J., \& Kingstone, A. (2000). Inhibition of return and visual search: How many separate loci are inhibited? Perception \& Psychophysics, 62, 452-458.

Wechsler, D. (1999). Wechsler abbreviated scale of intelligence. San Antonio: The Psychological Corporation.
Wright, R. D., \& Richard, C. M. (1996). Inhibition-of-return at multiple locations in visual space. Canadian Journal of Experimental Psychology, 50, 324-327. doi:10.1037/11961961.50.3.324

Yesavage, J. A., Brink, T. L., Rose, T. L., Lum, O., Huang, V., Adey, M., \& Leirar, V. D. (1982). Development and validation of a geriatric depression screening scale: A preliminary report. Journal of Psychiatric Research, 17, 37-49. doi:10.1016/0022-3956(82) 90033-4 